\title{
Movement automaticity as functional variability
}

\begin{abstract}
Functional variability has been suggested to reflect movement automaticity. To test this hypothesis, we used the Uncontrolled Manifold (UCM) approach to operationalize functional variability in darts. We gauged the association between movement automaticity and functional variability in four different ways. First, we investigated whether functional variability was higher in the second half of a throw than in the first half, because the duration of a darts throw is too short for conscious control to intervene. Second, we compared whether functional variability was higher in experts than in novices, because motor control is presumed to be more automatized in experts. Third, we manipulated conscious control via attentional focus instructions, presuming that internal focus instructions result in reduced automaticity, and thus may decrease functional variability. Fourth, we administered the Movement Specific Reinvestment Scale (MSRS), which estimates the propensity for conscious control and was therefore expected to be associated with functional variability. In line with the hypotheses, functional variability was higher in the second half than in the first half of darts throws. Furthermore, experts displayed more functional variability than novices. These results suggest that functional variability reflects movement automaticity. However, neither the focus manipulations, nor MSRS scores significantly predicted functional variability. Further study is therefore required to ascertain whether functional variability reflects movement automaticity.
\end{abstract}




\section{Introduction}

Theories about the role of cognition in motor performance often stress the importance of movement automaticity. For example, the theory of reinvestment (Masters \& Maxwell, 2008) warns that "automated motor processes can be disrupted if they are run using consciously accessed, task-relevant declarative knowledge" (p. 160). Similarly, the constrained action hypothesis (Wulf, 2013) holds that "an internal focus induces a conscious type of control, causing individuals to constrain their motor system by interfering with automatic control processes" (p. 91). The explicit monitoring theory (Beilock \& Carr, 2001) states that "the mechanisms of choking $^{1}$ operate on task control structures that are proceduralized - based on mental or motor programs that run largely unattended, without the services of working memory, and might best remain outside the scrutiny of introspection" (p. 701). These theories all agree that conscious control can adversely affect motor performance by reducing movement automaticity.

Since automaticity takes up such a prominent position in different theories, it is relevant to consider how it may be operationalized. In this respect, Wulf (2013) suggested that reductions in movement automaticity may be observed as disruptions of functional variability. Functional variability occurs when variability in some joints is compensated by appropriate variability in other joints (Scholz \& Schöner, 1999). Thereby, it helps to stabilize movement outcome in the face of movement variability. Furthermore, functional variability is said to represent the movement system's ability to regulate itself without relying on conscious adaptations (Müller \& Loosch, 1999). This goes especially for fast, ballistic movements. Given the fact that these movements provide insufficient time to allow for conscious control, the movement system is forced to

\footnotetext{
${ }^{1}$ Choking is "the occurrence of inferior performance despite striving and incentives for superior performance" (Baumeister \& Showers, 1986., p361)
} 
somehow regulate itself. It is therefore plausible that functional variability operationalizes movement automaticity in fast, ballistic movements.

Some studies report results consistent with Wulf's (2013) hypothesis. For instance, Lohse, Jones, Healy, and Sherwood (2014) found that an external focus - which promotes movement automaticity - was associated with more functional variability between arm joints in darts compared to an internal focus. Ford, Hodges, Huys, and Williams (2009) and Wulf and Dufek (2009) reported similar results in soccer and vertical jumping, respectively. Such findings suggest that functional variability may indeed reflect movement automaticity.

However, due to some issues, further scrutiny is required to ascertain the validity of this conclusion. For instance, opposing definitions of functional variability have been used. Whereas Lohse et al. (2014) define functional variability as high correlations between joint angles ${ }^{2}$, Ford et al. (2009) and Wulf and Dufek (2009) define it as low correlations between joint angles. Additionally, the measures used by Lohse et al. (2014) cover dart release (not the entire motion) and were sampled at a low rate of $30 \mathrm{~Hz}^{3}$, with joint positions identified manually. Therefore, it is hard to determine the reliability of the results.

This current study applied the uncontrolled manifold (UCM) approach (Scholz \& Schöner, 1999) to a darts task as a more valid measure of functional variability. The UCM method allows functional variability to be determined through all stages of a movement. Because the details of

\footnotetext{
${ }^{2}$ Lohse et al furthermore added that functional variability may be associated with an increase in overall variability.

${ }^{3}$ To compare, (darts) throwing studies (e.g. Aguinaldo \& Chambers, 2009; Müller \& Sternad, 2004; Nasu, Matsuo, \& Kadota, 2014; Smeets, Frens, \& Brenner, 2002; Wagner, Pfusterschmied, Klous, von Duvillard, \& Müller, 2012) commonly use sample frequencies of 250 to $700 \mathrm{~Hz}$.
} 
the UCM method will be explained in the methods section, it suffices here to state that such an approach can be used to quantify the degree of compensation between different joints to stabilise key determinants of performance outcome (Scholz \& Schöner, 1999), such as the magnitude and angle of velocity.

The current study examined whether functional variability is a valid operationalization of movement automaticity in four ways. First, functional variability was quantified in the first and the second half of the forward-motion of the dart throw. The first half was defined as half of the duration of the throw. The second half was defined as the remaining time. Any differences between these two phases are unlikely to be a product of conscious processes, as there is not enough time available to make conscious adaptations (Müller \& Loosch, 1999). The argument supporting this claim goes like this: Conscious control relies on neural feedback loops (e.g., transport of afferent signals, processing by the brain, and transport of efferent signals). These feedback loops take time. Fast, ballistic movements, such as darts throwing, are of short duration - i.e., shorter than the feedback loops involved in conscious control. Therefore, motor control, in these types of movements, cannot occur consciously. Consequently, differences in functional variability between the first and second half of a throw - i.e., motor control that occurs during a fast, ballistic movement - can be expected to reflect automatized processes.

A second way of examining whether functional variability reflects movement automaticity was to compared experts and novices. In line with previous studies (Button, Macleod, Sanders, \& Coleman, 2003; Müller \& Loosch, 1999), we predicted that experts - whose movements should be more automatized (Fitts \& Posner, 1967) - would display more functional variability than novices. Third, conscious control - and therefore movement automaticity - was manipulated by focus of attention instructions adapted from Lohse et al. (2014). In line with Lohse et al. (2014), we 
expected that an external focus of attention would increase automaticity and thus lead to more functional variability than an internal focus. Fourth, we determined individual's propensity for conscious control (Malhotra, Poolton, Wilson, Ngo, \& Masters, 2012; Masters \& Maxwell, 2008) using the Movement Specific Reinvestment Scale (MSRS) (Masters, Eves, \& Maxwell, 2005). The MSRS estimates the likelihood that participants will adopt conscious control, and conversely, their propensity to rely on movement automaticity. Participants with a low propensity for conscious control - and therefore a high propensity for movement automaticity - were expected to show higher levels of functional variability than those with a high propensity for conscious control.

\section{Methods}

The study protocol was approved by the Ethical Committee of the University at which the experiment was conducted.

Participants. Twenty participants $(8$ male, 12 female; mean age $=31.5$ years; $S D=8.7$ ) took part in this experiment. All had normal or corrected to normal vision. Participants consisted of 14 novices who had no experience of playing competitive darts and did not practice, and 6 experts who had an average of $14.2(S D=7.4)$ years of experience playing competitive darts and spent an average of $5.3(S D=2.5)$ hours per week playing darts. Participants were compensated 10 Euros for their participation.

Apparatus. A standard sized dartboard was used. The board was positioned at standard height $(1.73 \mathrm{~m})$ and distance $(2.37 \mathrm{~m})$. A digital video camera captured landing positions of the darts on the board. Participants were allowed to use their own darts. Alternatively, a set of standard size and weight, tungsten, steel tip darts were provided. An active, optoelectronic, infra-red camera 
system was used to record participants' throwing motion (Optotrak 3020, Northern Digital Inc, Waterloo Ontario). The camera system recorded marker displacement in 3 dimensions at a frequency of $500 \mathrm{~Hz}$. Markers were placed on the torso (sternum), shoulder (dorsal ${ }^{4}$ part or the deltoid muscle), elbow (medial epicondyle) process), wrist (ulnar styloid) and on the nail of the thumb. To ensure marker visibility, participants wore a sleeveless shirt. A Dutch version (Kal et al., 2016) of the MSRS (Masters et al., 2005) was used to record participants' propensity for conscious control. The MSRS is a 10-item questionnaire requiring responses on a 6-point Likert scale that ranges from "strongly disagree" to "strongly agree". The scale comprises a Conscious Motor Processing (CMP) and a Movement Self-Consciousness (MS-C) subscale. The subtle difference between these subscales is that MS-C captures the propensity for conscious attention to movement, whereas CMP captures the propensity to make conscious attempts to interfere with ongoing movements (Malhotra, Poolton, Wilson, Fan, \& Masters, 2014; Malhotra et al., 2012; Masters \& Maxwell, 2008; Van Ginneken et al., 2017).

Procedure. After participants signed informed consent and were familiarized with the equipment, they were fitted with optoelectric markers. During a brief warm-up, participants completed two blocks of 9 practice throws separated by a block of 3 throws with an internal focus instruction and a block of 3 throws with an external focus instruction to familiarize themselves with the instructions. The focus of attention instructions were identical to instructions used by Van Ginneken et al. (2017) who had based them on Lohse, Sherwood, and Healy (2010). The internal focus instructions were: "Please visually focus on the target and mentally focus on the movements of your arm. Think of how your arm should move". The external focus instructions were: "Please

\footnotetext{
${ }^{4}$ The reason that this was not a bony landmark was to enable all markers (including the thumb) to be recorded from a single vantage point. As this vantage point was frontal to the thorax, no bony landmark on the shoulder was available.
} 
visually focus on the target and mentally focus on the flight of the dart. Think of how the dart should fly". Participants were instructed to always aim for the bulls-eye of the dartboard. After completing the warm-up, participants engaged in two blocks of 45 throws in which the same focus of attention instructions were provided as during the warm-up (one set of instructions in each block). The instructions were repeated verbally, in a neutral voice, after every $6^{\text {th }}$ throw (i.e., after every 2 sets of 3 darts thrown) as the participants were collecting the darts from the board. The order of internal and external foci was randomized. Finally, participants completed the MSRS, were debriefed and compensated 10 Euros for their participation.

\section{Measures.}

Throwing Accuracy. Although not directly related to the goal of this study, we did take throwing accuracy into account. It served three functions. First, it was a way to ascertain whether the experts were indeed better darts players than the novices. Second, it was a manipulation check for our attentional focus manipulation. Third, it was a means to determine criterion validity of the MSRS scale. This is possible, because the three aforementioned theories (Beilock \& Carr, 2001; Masters \& Maxwell, 2008; Wulf, 2013) all propose an association between conscious control and motor performance. As the MSRS scale is proposed to operationalize propensity for conscious control (Masters et al., 2005), its criterion validity can be tested through motor performance.

Throwing accuracy was determined by the vertical mean distance error (MDE) of the landing position of the darts on the board. This was done to make throwing accuracy and functional variability more comparable. In darts, movements primarily take place in a vertical direction and in the direction of the target. Although, sideways motion is possible, it is hard to identify two or more joints that may compensate each other's sideways movements. Furthermore, because lateral 
error tends to be substantially smaller than vertical error (Tibshani, Price \& Taylor, 2011). The latter may therefore be ignored (e.g., Smeets, Frens \& Brenner, 2002). Therefore, sideways motion and throwing error were ignored.

Functional variability. All kinematic and video data was processed using Matlab version R2012a. A $2^{\text {nd }}$ order Butterworth low-pass filter with a cutoff value of $10 \mathrm{~Hz}$ was applied to the displacement data of the markers, which preserved more than $99 \%$ of the signal. It was assumed that displacement of the dart was identical to that of the thumb. Each throw was analyzed from the beginning of the forward-motion to the moment of release. The moment of release was determined as follows: First, for each frame a hypothetical landing position on the dart board was determined based on velocity, angle and position of the thumb and assuming frictionless flight. Subsequently, the frame that showed the highest similarity between the hypothetical and the actual landing position of the dart was identified as the frame in which the dart must have been released ${ }^{5}$. The start of the forward-motion was defined as the moment that the thumb changed velocity - i.e. a change from moving away from the target to moving towards it. Torso orientation and shoulder, elbow and wrist angles were determined based on displacement data of the markers.

To quantify functional variability, the UCM approach (Latash, Scholz, \& Schöner, 2007; Martin, Scholz, \& Schöner, 2009; Scholz \& Schöner, 1999) was applied. After time normalizing all markers positions between the beginning of the forward-motion and dart-release, we examined to what degree elemental variables (i.e. the aforementioned joint angles) worked together to stabilize different performance variables ${ }^{6}$ during a throw.

\footnotetext{
${ }^{5}$ Although this method is novel, it is sensible and captures a standard moment in darts throws corresponding with the moment of release.

${ }^{6}$ The term performance variable does not directly refer to performance outcome, but key determinants of the outcome.
} 
In real-world tasks, such as darts, it can be challenging to obtain valid measures of functional variability. It is often hard (or even impossible) to select appropriate performance variables (Latash, 2008) and levels of functional variability may differ depending on which reference frame is chosen to express the data (Smeets \& Louw, 2007). To deal with this issue, we selected multiple performance variables and observed them using different reference frames. Although we could not predict which performance variables would be stabilized, we did know which variables determine throwing accuracy. As dart flights are ballistic, vertical error is fully determined by the magnitude and angle of velocity, and the horizontal position (in the direction towards the dart board, not sideways) and vertical position of the darts at release. Therefore, we used these 4 performance variables. Each variable was determined twice: once using the bulls eye as the origin of the reference frame and once using the shoulder marker as the origin. The joint angles were used as elemental variables.

The degree to which the elemental variables stabilized each performance variable was captured by calculation of an index of synergy (IOS). This method breaks down the total variance in all joints $\left(\mathrm{V}_{\mathrm{T}}\right.$ ) into two components; one component representing the (co-)variation that stabilizes a performance variable $\left(\mathrm{V}_{\mathrm{UCM}}\right)$ - i.e. variability in some joints that is compensated by appropriate variability in others - and one component representing the variability that destabilizes the performance variable $\left(\mathrm{V}_{\mathrm{ORT}}\right)$. IOS values were normalized, in line with Krishnan, Rosenblatt, Latash, and Grabiner (2013), using a Fisher's Z transformation, such that IOS $=0.5 \cdot \log [(\mathrm{n}+$ $\left.\left.\mathrm{V}_{\mathrm{Z}}\right) /\left((\mathrm{n} / \mathrm{d})-\mathrm{V}_{\mathrm{Z}}\right)\right]$, whereby $\mathrm{V}_{\mathrm{Z}}=\left(\mathrm{V}_{\mathrm{UCM}}-\mathrm{V}_{\mathrm{ORT}}\right) / \mathrm{V}_{\mathrm{TOT}}$ and $\mathrm{n}$ is the number of elemental variables and $d$ is the number of dimensions of the performance variable. IOS values were calculated for the first and the second half of the forward-motion of the darts throws. 
The presence of a synergy is indicated if $\mathrm{V}_{\mathrm{UCM}}$ is bigger than $\mathrm{V}_{\text {ORT. }}$. As stated by Krishnan et al. (2013) and Robert, Bennett, Russell, Zirker, and Abel (2009), $\mathrm{V}_{\mathrm{UCM}}>\mathrm{V}_{\mathrm{ORT}}$ when IOS $>0.5$ $* \log [(\mathrm{n} / \mathrm{d}) /(\mathrm{n} /(\mathrm{n}-\mathrm{d}))]$. In this study, we used 4 elemental variables and each performance variable had 1 dimension. Therefore, the cutoff value for when a synergy could be considered to be present was .24 (i.e. $0.5 * \log [(4 / 1) /(4 /(4-1))]=0.5 * \log [3]=.24)$. Only the performance variables for which synergies were present were used for further statistical analyses.

Data Analysis. All statistical analyses were performed using IBM SPSS Statistics version 21. Cronbach's Alpha was used to determine the internal consistency of the MSRS and its two subscales. A Pearson correlation coefficient was calculated to evaluate the divergent validity of the two subscales. Generalized Linear Mixed Models (GLMM) were used to investigate the effects of forward-motion phase (first half / second half), expertise (experts / novices), focus of attention (internal / external), propensity for conscious control (MSRS score), reference frame origin (bullseye / shoulder marker), performance variable (velocity magnitude / velocity angle / horizontal position / vertical position) and foci-order (internal first group / external first group) on IOS. The GLMM for MDE only tested for the effects of expertise, focus of attention, propensity for conscious control and foci-order. All main effects, two-way and three-way interactions were taken into account as fixed factors. Furthermore, inter-individual differences in overall performance were taken into account as random factors, thereby controlling for the correlation between repeated measures that is expected to occur due to random variation in inter-individual differences in performance. The method for controlling for inter-individual difference was autoregressive AR-1.

Similar to stepwise linear regressions, GLMM first evaluates whether the inclusion independent variable (or interaction) as fixed factor improves the overall fit of a model based on Aikake's Information Criterion. If it does, then it is included in the model. If it does not, it is 
excluded. Only when a variable is included in the model, is its significance tested for. Effects of included variables may still turn out to be non-significant. A median split was used to follow up on significant effects of MSRS scores. The Bonferroni correction was applied when multiple comparisons were made to follow up significant effects of performance variable.

\section{Results}

Descriptive statistics. Descriptive statistics for the effects of all independent variables on IOS and MDE can be found in Table 1 and Table 2, respectively. The results of IOS show that on average there were synergies for all performance variables, as they all surpassed the cutoff value of .24. All performance variables were therefore included in the subsequent statistical tests.

\begin{tabular}{|c|c|c|c|}
\hline Variable & Levels & M IOS & SD IOS \\
\hline \multirow{4}{*}{ Expertise } & Experts & .56 & .38 \\
& & .36 & .32 \\
\cline { 2 - 4 } & Novices & .43 & .29 \\
\hline \multirow{2}{*}{ Focus of attention } & External & .41 & .37 \\
\cline { 2 - 4 } & Internal & .41 & .38 \\
\hline \multirow{2}{*}{$\begin{array}{c}\text { Propensity for } \\
\text { conscious control }\end{array}$} & Low propensity & .43 & .28 \\
\cline { 2 - 4 } & High propensity & .42 & .34 \\
\hline Foci-order & External first group & .43 & .36 \\
\cline { 2 - 4 } & Internal first group & .37 & .39 \\
\hline \multirow{2}{*}{$\begin{array}{c}\text { Forward-motion } \\
\text { phase }\end{array}$} & First half & .47 & .30 \\
\cline { 2 - 4 } & Second half & .46 & .37 \\
\hline Reference frame & Bulls-eye & .38 & .31 \\
\cline { 2 - 4 } & Shoulder marker & .31 & .28 \\
\hline Performance variable & Velocity magnitude & .53 & .35 \\
\cline { 2 - 4 } & Velocity angle & .50 & .39 \\
\cline { 2 - 4 } & Horizontal position & .35 & \\
\cline { 2 - 4 } & Vertical position & & \\
\hline
\end{tabular}

Table 1. Descriptive statistics for all independent variables regarding IOS. 


\begin{tabular}{|c|c|c|c|}
\hline \multirow{2}{*}{ Variable } & Levels & M MDE & SD MDE \\
\hline \multirow{2}{*}{ Expertise } & Experts & .91 & .27 \\
\cline { 2 - 4 } & Novices & 2.84 & .86 \\
\hline Focus of attention & External & 2.17 & 1.31 \\
\cline { 2 - 4 } & Internal & 2.24 & 1.25 \\
\hline \multirow{2}{*}{$\begin{array}{c}\text { Propensity for } \\
\text { conscious control }\end{array}$} & Low propensity & 1.94 & .97 \\
\cline { 2 - 4 } & High propensity & 2.74 & 1.29 \\
\hline Foci-order & External first group & 2.43 & .81 \\
\cline { 2 - 4 } & Internal first group & 2.06 & \\
\hline
\end{tabular}

Table 2. Descriptive statistics for all independent variables regarding MDE.

MSRS. The internal consistency of the MSRS was acceptable to good $(\alpha=.846)$ as it was for the MS-C ( $\alpha=.762)$ and CMP ( $\alpha=.706)$ subscales. However, the subscales were highly correlated $(r=.65, p<.01)$. We therefore collapsed both scores into a total MSRS score. Furthermore, experts scored significantly higher on the MSRS scale $(M=28.33, S D=6.25)$ than novices $(M=18.93, S D=8.19)$. To prevent confounding between the effects of expertise and MSRS scores, we centered the MSRS scores within the group of experts and within the group of novices by subtracting the respective group means from the individual MSRS scores.

Throwing accuracy. The best fitting model predicting MDE included the main effects of expertise $(F(1,632)=1130.79, p<.001)$, focus of attention $(F(1,632)=.40, p=.53)$, propensity for conscious control $(F(1,632)=6.91, p<.05)$, and foci-order $(F(1,632)=.85, p=.46)$ and the interactions between expertise and focus of attention $(F(1,632)=.09, p=.76)$, focus of attention and propensity for conscious control $(F(1,632)=.71, p=.40)$, and focus of attention and foci$\operatorname{order}(F(1,632)=21.10, p<.001)$. 
The main effect of expertise showed that experts $(M=.91, S D=.27)$ displayed significantly lower MDE than novices $(M=2.84 S D=.86),(t(632)=-24.53, p<.001,95 \% C I=[-1.80-1.53])$. The main effect of propensity for conscious control suggested that participants with a low propensity for conscious control had a lower $\operatorname{MDE}(M=1.95, S D=1.03)$ than participants with a high propensity $(M=2.57, S D=1.24),(t(632)=-3.08, p<.01,95 \% C I=[-.32-.07])$. Further exploration of the interaction between focus of attention and foci-order revealed that in the first block participants who adopted an internal focus $(M=2.05, S D=.80)$ were more accurate than those who adopted an external focus $(M=2.38, S D=1.41),(t(314)=-5.80, p<.001,95 \% C I=$ [-.70 -.35]). In the second block however, those who adopted an internal focus $(M=2.45, S D=$ 1.37) were less accurate than those who adopted an external focus $(M=2.07, S D=.82),(t(314)=$ $7.09, p<.001,95 \% C I=[.40 .72])$.

Functional variability. The best fitting model predicting IOS included the main effects of forward-motion phase $(F(1,629)=80.79, p<.001)$, expertise $(F(1,629)=61.42, p<.001)$, focus of attention $(F(1,629)=.01, p=.94)$, propensity for conscious control $(F(1,629)=.23, p=.63)$, reference frame origin $(F(1,629)=46.37, p<.001)$, performance variable $(F(1,629)=20.69, p$ $<.001)$ and foci-order $(F(1,629)=2.00, p=.16)$ and the interaction between expertise and reference frame origin $(F(1,629)=30.95, p<.001)$.

The main effect of forward-motion phase showed that in the first half of the throw $(M=.37$, $S D=.39)$ IOS was lower $(t(629)=8.90, p<.001,95 \% C I=[.15 .23])$ than in the second half $(M$ $=.47, S D=.30)$. The main effect of expertise showed that experts $(M=.56, S D=.38)$ displayed significantly higher IOS than novices $(M=.36, S D=.32),(t(629)=8.81, p<.001,95 \% C I=$ $\left[\begin{array}{ll}23 & 36]) .\end{array}\right.$ The main effect of reference frame origin revealed that IOS was significantly lower when the bulls-eye was used as the origin of the reference frames $(M=.38, S D=.32)$ than when 
the shoulder marker $(M=.46, S D=.37),(t(629)=-4.29, p<.001,95 \% C I=[-.14-.05])$ was used. The main effect of performance variable revealed that the horizontal position $(M=.50, S D=.35)$ and angle $(M=.53, S D=.30)$ of the thumb marker showed significantly higher IOS than its vertical position $(M=.35, S D=.39)$ and velocity $(M=.31, S D=.31),(p s<.01)$. The interaction between expertise and reference frame origin showed that the experts $(M=.70, S D=.39)$ displayed a higher IOS than novices $(M=.37, S D=.37)$ when the shoulder marker was used as the origin of the reference frame $(t(311)=9.61, p<.001,95 \% C I=[.25 .38])$. By contrast, when the bulls-eye was used as the origin, there were no significant differences between experts $(M=.43, S D=.32)$ and novices $(M=.36, S D=.32),(t(311)=1.65, p=.10,95 \% C I=[-.01 .10])$.

\section{Discussion}

This study investigated whether functional variability provides a valid operationalization of movement automaticity. Functional variability supports motor performance by preserving consistency of movement outcomes despite the presence of movement variability. It does so by compensating variability in some joints by appropriate variability in others. As darts throws are of short duration, it is unlikely that these compensations are the product of slow, conscious control processes. Therefore, it was expected that functional variability reflected movement automaticity.

Experts and novices engaged in a dart-throwing task in which they were instructed to adopt internal and external foci of attention in different blocks of 45 throws. Participants also completed the MSRS (Masters et al., 2005) to determine their propensity for conscious control. Functional variability was determined using the UCM method (Scholz \& Schoner, 1999), because it quantifies how variability in different joints co-varies to stabilize the outcome of key determinants of motor performance. This was done in the first and second half of darts throws to investigate whether 
functional variability increases during a throw outside the influence of conscious control for which there is not enough time available (Müller \& Loosch, 1999). Throwing accuracy - defined as vertical mean distance error (MDE) relative to the bulls-eye - was analysed to ascertain whether the experts were better than the novices. It also served as a manipulation check of the attentional focus manipulation and to ascertain criterion validity of the MSRS scale.

Throwing accuracy revealed mixed results. Unsurprisingly, experts threw more accurately than the novices, confirming that the experts were indeed better darts players than the novices. Furthermore, in line with the theory of reinvestment (Masters \& Maxwell, 2008), constrained action hypothesis (Wulf, 2013) and explicit monitoring theory (Beilock \& Carr, 2001), conscious control propensity was negatively related to throwing accuracy. This provided confidence in the criterion validity of the MSRS scale. However, the absence of an effect of our attentional focus manipulation indicates that, in contrast to the studies by Lohse et al. (2014) and Van Ginneken et al. (2017), the focus instructions failed to produce their expected effects. Conscious control - and therefore movement automaticity - appeared to have influenced throwing accuracy in some, but not all respects.

One anomaly occurred. Even though we had randomized which attentional focus instruction participants encountered first, those presented with the internal instruction in the first block of 45 throws outperformed those presented with the external instruction first. They did so in the first block as well as in the second block, indicating that random allocation did not result in groups of equal throwing accuracy. Instead, one randomly allocated group of participants just so happened to throw more accurately than the other, even though the number of experts was equal in both groups $(n=3)$. It is unclear whether this anomaly influenced the effects of any other variables, but there is an off chance that it may have interfered with the effect of the attentional 
focus manipulation.

There was partial evidence for the assertion that functional variability operationalizes movement automaticity. Functional variability was higher in the second half of the throws than in the first half. It is unlikely that participants had enough time to make conscious adaptations over the course of the forward-motion of the throw (Müller \& Loosch, 1999), which typically lasted between 100 and 200 milliseconds. The increase in functional variability is therefore likely to reflect automatic processes. Furthermore, experts also showed overall greater functional variability than novices, replicating the results of previous studies that found that the difference in automaticity between experts and novices surfaces as a difference in functional variability (Button et al., 2003; Müller \& Loosch, 1999). Taken together, these findings support the assertion that functional variability reflects movement automaticity.

Interestingly, experts only showed more functional variability than novices when the shoulder marker was used as origin of the reference frame, but not when the bulls-eye was used. This interaction also resulted in a main effect of reference frame origin, whereby functional variability was higher when the shoulder marker was used as reference frame origin. As we deemed possible, different reference frames indeed lead to different results regarding functional variability. It is hard to conjure up a reliable explanation for these results. However, it may be speculated that an internal reference frame is more sensitive to the interplay of forces between joints that may partially govern functional variability. To get a sense of how this interplay might work, imagine an unplanned - say due to noise - slowing down of the angular velocity of the elbow. Mechanical laws of motion prescribe that this slowing down of the elbow causes a momentum in the wrist that prompts it to accelerate. Due to this inherent relationship between elbow and wrist, the position and velocity of the darts are automatically stabilized. Experts may be particularly apt at exploiting 
this joint interplay to their advantage, thereby quite literally automatizing their motor control. Furthermore, a reference frame outside a person's body (e.g. the bulls-eye) picks up on the joint interplay as well as on differences in body posture (e.g. forward lean), while a reference frame on the body (e.g. the shoulder marker) is more selectively sensitive to the joint interplay. Therefore, the two reference frames may have resulted in different results regarding functional variability.

As we mentioned, our results were inconclusive. Contrary to the hypotheses, focus of attention and propensity for conscious control did not significantly predict functional variability. Several speculations may explain these mixed results. For example, the attentional focus instructions may not have successfully manipulated conscious control / movement automaticity. This was already indicated by the absence of an effect of attentional focus on throwing accuracy. The lab setting, which included multiple cameras and the placement of markers on the participants' limbs, may for example have induced high levels of conscious control (and therefore low levels of movement automaticity) in all participants. Alternatively, although the MSRS captures predispositions for conscious control, it does not guarantee to predict actual levels of conscious control in specific situations. It is for example possible that our focus manipulations washed out the effect of personality predispositions. Whereas normally some people may have been more inclined than others to adopt conscious control, in the current study they may all have simply done so in accordance with the degree to which they were instructed to do so. Furthermore, the fact that experts scored higher on the MSRS than novices might have obfuscated some of its effects.

Alternatively, the absence of effects of focus of attention and propensity of conscious control on functional variability may call into question a tacit assumption regarding the relationship between conscious control and movement automaticity. It is generally assumed that conscious control and movement automaticity are two ends of a continuum, such that changes in 
conscious control are inherently associated with opposing changes in movement automaticity. However, Bernstein (1996) identifies different aspects of skilled motor performance - i.e. adaptability, manoeuvrability and resourcefulness - which in turn may be governed consciously as well as in automatized fashion. Distinguishing between conscious control and movement automaticity may therefore not be as straightforward as is sometimes assumed.

The difficulty to distinguish between conscious control and movement automaticity is further demonstrated by a practical issue. In this study, we assumed based on argumentations by Müller and Loosch (1999) that conscious control would have little chance to influence functional variability during a dart throw. Although this assumption is reasonable, it ignores the fact that the UCM approach determines functional variability based on comparisons across trials. It is therefore sensitive to adaptations during darts throws as well as - possibly conscious - strategic changes between trials. It is therefore not entirely certain whether between-trial functional variability can successfully distinguish between conscious control and movement automaticity.

It is apparent that further study is required to ascertain movement automaticity can be operationalized as functional variability. These studies may include further manipulations of conscious control, such as psychological and time pressure. Secondary tasks or probe reaction tasks may be used to gauge levels of conscious control. Furthermore, within-trial functional variability (Scholz, Kang, Patterson, \& Latash, 2003) may be better suitable than between-trial functional variability to isolate movement variability. Lastly, we would like to point out that the UCM approach provides an overall measure of the degree to which multiple elemental variables stabilize performance variables. It may be of interest to further investigate exactly which elemental variables contribute more than others under different conditions. Such efforts may help to shed more light on the - in this study partially supported - assertion that functional variability 
operationalizes movement automaticity.

\section{References}

Aguinaldo, A. L., \& Chambers, H. (2009). Correlation of throwing mechanics with elbow valgus load in adult baseball pitchers. The American journal of sports medicine, 37(10), 20432048. doi:10.1177/0363546509336721

Beilock, S. L., \& Carr, T. H. (2001). On the fragility of skilled performance: What governs choking under pressure? Journal of experimental psychology: General, 130(4), 701-725. doi:10.1037/0096-3445.130.4.701

Bernstein, N. A. (1996). Dexterity and its development. Hove, United Kingdom: Psychology Press.

Button, C., Macleod, M., Sanders, R., \& Coleman, S. (2003). Examining movement variability in the basketball free-throw action at different skill levels. Research quarterly for exercise and sport, 74(3), 257-269. doi:10.1080/02701367.2003.10609090

Fitts, P. M., \& Posner, M. I. (1967). Human performance. Belmont, CA: Brooks/Cole.

Ford, P., Hodges, N. J., Huys, R., \& Williams, A. M. (2009). An evaluation of end-point trajectory planning during skilled kicking. Motor control, 13(1), 1-24. doi:/10.1123/mcj.13.1.1

Kal, E., Houdijk, H., Van der Wurff, P., Groet, E., Van Bennekom, C., Scherder, E., \& Van der Kamp, J. (2016). The inclination for conscious motor control after stroke: Validating the Movement-Specific Reinvestment Scale for use in inpatient stroke patients. Disability and rehabilitation, 38(11), 1097-1106. doi:10.3109/09638288.2015.1091858

Krishnan, V., Rosenblatt, N. J., Latash, M. L., \& Grabiner, M. D. (2013). The effects of age on stabilization of the mediolateral trajectory of the swing foot. Gait \& posture, 38(4), 923928. doi:10.1016/j.gaitpost.2013.04.023

Latash, M. L. (2008). Synergy: Oxford University Press. 
Latash, M. L., Scholz, J. P., \& Schöner, G. (2007). Toward a new theory of motor synergies. Motor Control, 11(3), 276. doi:10.1123/mcj.11.3.276

Lohse, K. R., Jones, M., Healy, A. F., \& Sherwood, D. E. (2014). The role of attention in motor control. Journal of Experimental Psychology: General, 143(2), 930-948. doi:10.1037/a0032817

Lohse, K. R., Sherwood, D. E., \& Healy, A. F. (2010). How changing the focus of attention affects performance, kinematics, and electromyography in dart throwing. Human Movement Science, 29(4), 542-555. doi:10.1016/j.humov.2010.05.001

Malhotra, N., Poolton, J. M., Wilson, M. R., Fan, J. K. M., \& Masters, R. S. W. (2014). Conscious Motor Processing and Movement Self-Consciousness: Two Dimensions of Personality That Influence Laparoscopic Training. Journal of Surgical Education, 71(6), 798 - 804. doi:10.1016/j.jsurg.2014.04.003

Malhotra, N., Poolton, J. M., Wilson, M. R., Ngo, K., \& Masters, R. S. W. (2012). Conscious monitoring and control (reinvestment) in surgical performance under pressure. Surgical endoscopy, 26(9), 2423-2429. doi:10.1007/s00464-012-2193-8

Martin, V., Scholz, J. P., \& Schöner, G. (2009). Redundancy, self-motion, and motor control. Neural Computation, 21(5), 1371-1414. doi:10.1162/neco.2008.01-08-698

Masters, R. S. W., Eves, F. F., \& Maxwell, J. P. (2005). Development of a movement specific reinvestment scale. Paper presented at the ISSP 11th World Congress of Sport Psychology, Sydney, Australia.

Masters, R. S. W., \& Maxwell, J. P. (2008). The theory of reinvestment. International Review of Sport and Exercise Psychology, 1(2), 160-183. doi:10.1080/17509840802287218 
Müller, H., \& Loosch, E. (1999). Functional variability and an equifinal path of movement during targeted throwing. Journal of Human Movement Studies, 36, 103-126.

Müller, H., \& Sternad, D. (2004). Decomposition of variability in the execution of goal-oriented tasks: Three components of skill improvement. Journal of Experimental Psychology: Human Perception and Performance, 30(1), 212-233. doi:10.1037/0096-1523.30.1.212

Nasu, D., Matsuo, T., \& Kadota, K. (2014). Two types of motor strategy for accurate dart throwing. PloS one, 9(2), e88536.

Robert, T., Bennett, B. C., Russell, S. D., Zirker, C. A., \& Abel, M. F. (2009). Angular momentum synergies during walking. Experimental brain research, 197(2), 185-197. doi:10.1007/s00221-009-1904-4

Scholz, J. P., Kang, N., Patterson, D., \& Latash, M. L. (2003). Uncontrolled manifold analysis of single trials during multi-finger force production by persons with and without Down syndrome. Experimental Brain Research, 153(1), 45-58. doi:10.1007/s00221-003-1580-8

Scholz, J. P., \& Schöner, G. (1999). The uncontrolled manifold concept: Identifying control variables for a functional task. Experimental brain research, 126(3), 289-306. doi:10.1007/s002210050738

Smeets, J. B., Frens, M. A., \& Brenner, E. (2002). Throwing darts: Timing is not the limiting factor. Experimental Brain Research, 144(2), 268-274. doi:10.1007/s00221-002-1072-2

Smeets, J. B., \& Louw, S. (2007). The contribution of covariation to skill improvement is an ambiguous measure: Comment on Muller and Sternad (2004). Journal of Experimental Psychology: Human Perception and Performance, 33(1), 246-249; discussion 250-245. doi:10.1037/0096-1523.33.1.246 
Tibshani, R. J., Price, A., \& Taylor, J. (2011). A statistician plays darts. Journal of the Royal Statistical Society, 174, 1, 213 - 226.

Van Ginneken, W. F., Poolton, J. M., Masters, R. S. W., Capio, C. M., Kal, E. C., \& van der Kamp, J. (2017). Comparing the effects of conscious monitoring and conscious control on motor performance. Psychology of Sport and Exercise, 30, 145-152. doi:10.1016/j.psychsport.2017.03.001

Wagner, H., Pfusterschmied, J., Klous, M., von Duvillard, S. P., \& Müller, E. (2012). Movement variability and skill level of various throwing techniques. Human movement science, 31(1), 78-90. doi:10.1016/j.humov.2011.05.005

Wulf, G. (2013). Attentional focus and motor learning: a review of 15 years. International Review of Sport and Exercise Psychology, 6(1), 77-104. doi:10.1080/1750984x.2012.723728

Wulf, G., \& Dufek, J. S. (2009). Increased jump height with an external focus due to enhanced lower extremity joint kinetics. Journal of Motor Behavior, 41(5), 401-409. doi:10.1080/00222890903228421 